\title{
Luminescence properties of piezoelectric single crystals with langasite structure
}

\author{
Minoru Itoh $^{\mathrm{a} *}$, Shinya Takagi ${ }^{\mathrm{a}}$, Mamoru Kitaura ${ }^{\mathrm{b}}$, Masami Fujita ${ }^{\mathrm{c}}$, Naoyuki Endo ${ }^{\mathrm{d}}$ \\ a Department of Electrical and Electronic Engineering, Shinshu University, \\ Nagano 380-8553, Japan \\ ${ }^{\mathrm{b}}$ Fukui National College of Technology, Sabae 916-8507, Japan \\ ' Japan Coast Guard Academy, Kure 737-8512, Japan \\ ${ }^{d}$ Cimeo Precision Co., Ltd., Miyota 389-0295, Japan
}

\begin{abstract}
Luminescence properties of single crystals of langasite (LGS), langataite (LGT), and langanite (LGN) are investigated at $5 \mathrm{~K}$ by using synchrotron radiation as an exciting light source. Two emission bands are observed at 420 and $500 \mathrm{~nm}$ in LGS, at 410 and $460 \mathrm{~nm}$ in LGT, and at 500 and $535 \mathrm{~nm}$ in LGN. The origin of these bands is discussed by reference to the electronic structure of LGS.
\end{abstract}

PACS Codes: 71.35.--y, 77.84.-s, 78.55.Hx

Keywords: Photoluminescence; Self-trapped exciton; Langasite; Langataite; Langanite

*Corresponding author: Minoru Itoh; Department of Electrical and Electronic Engineering, Faculty of Engineering, Shinshu University, Nagano 380-8553, Japan; Tel: +81-26-269-5261; Fax: +81-26-269-5220; E-mail: itohlab@shinshu-u.ac.jp 


\section{Introduction}

The langasite family crystals belong to the trigonal system with the space group P321, and are isostructural to $\mathrm{Ca}_{3} \mathrm{Ga}_{2} \mathrm{Ge}_{4} \mathrm{O}_{14}$ [1]. They consist of tetrahedron layers, perpendicular to the $c$-axis, between which there are layers formed by cations in distorted cubes coordinated by eight $\mathrm{O}$ atoms and in octahedra by six $\mathrm{O}$ atoms. Langasite $\left(\mathrm{La}_{3} \mathrm{Ga}_{5} \mathrm{SiO}_{14}\right.$; LGS) is a typical crystal of this family. Langataite $\left(\mathrm{La}_{3} \mathrm{Ga}_{5.5} \mathrm{Ta}_{0.5} \mathrm{O}_{14}\right.$; LGT) and langanite $\left(\mathrm{La}_{3} \mathrm{Ga}_{5.5} \mathrm{Nb}_{0.5} \mathrm{O}_{14}\right.$; LGN) are obtained by substituting $\mathrm{Si}^{4+}$ of LGS with $\mathrm{Ga}^{3+}, \mathrm{Ta}^{5+}$ and $\mathrm{Ga}^{3+}, \mathrm{Nb}^{5+}$, respectively. These crystals have electromechanical and acoustic characteristics attractive for application [2,3]; for instance, their piezoelectric moduli are 3-5 times as large as that of quartz.

Because of industrial concerns for langasite family, there are some papers on the crystal growth and acousto-electronic properties [3,4]. However, the fundamental optical characteristics have not been studied, except for our recent work on LGS [5]. In the present paper, we focus our attention on luminescence properties of LGS, LGT, and LGN crystals.

\section{Experiment}

Single crystals of LGS, LGT, and LGN were obtained from Mitsubishi Materials (Japan), Fomos (Russia), and Vniisms (Russia), respectively. These crystals were grown by the Czochralski method, and annealed at $1000-1300{ }^{\circ} \mathrm{C}$ for more than $24 \mathrm{~h}$ in reduction atmosphere. The crystals of LGS were completely transparent, while those of LGT and LGN were transparent but exhibited a slight amber color. The coloration may be linked to the existence of excess oxygen in the crystals. The crystal orientation was confirmed by an X-ray diffraction analysis. The square surfaces of samples, perpendicular to the $X$ - and $Y$-axes, were mechanically polished.

The present experiments were performed with use of synchrotron radiation from the UVSOR storage ring in the Institute for Molecular Science, Okazaki. For measurements of the polarization dependence, we used two samples with different orientations; one has the $Y$-axis parallel to the electric vector of the incident light $(E / / Y)$ and the other has the $Z$-axis $(E / / Z)$. Luminescence from the sample surface was dispersed through an Acton SpectraPro-3000i monochromator equipped with an LN/CCD camera (Princeton, 1100 PBVISAR). The excitation wavelength $\lambda_{\mathrm{ex}}$ was changed step by step, and for each step the emission spectrum was measured, so that we could get full information of excitation-emission spectra in a short time. The emission spectra were not corrected for the spectral response of the detection system, while the excitation spectra were corrected for the intensity distribution of the incident light.

\section{Results}


The transmission spectra were examined for $E / / Y$ and $E / / Z$ at $8 \mathrm{~K}$. It was found that the cut-off wavelengths of LGS, LGT, and LGN are independent of the polarization of the incident light, and are 235, 242, and $284 \mathrm{~nm}$, respectively.

Three-dimensional (3D) display of the excitation-emission spectrum of LGS measured for $E / / Y$ at $5 \mathrm{~K}$ is shown in Fig. 1 . One can see two emission bands at 420 and $500 \mathrm{~nm}$. The intensity of the 420-nm band starts to increase at $\lambda_{\mathrm{ex}}=220 \mathrm{~nm}$, and reaches the maximum around $205 \mathrm{~nm}$, followed by the decrease in the shorter wavelength region. On the other hand, the 500-nm band is intensely stimulated with UV light at $\lambda_{\mathrm{ex}}=245 \mathrm{~nm}$. The result obtained for $E / / Z$ was almost the same as Fig. 1.

The 3D spectrum of LGT for $E / / Y$ is shown in Fig. 2. Two emission bands are seen at 410 and $460 \mathrm{~nm}$. The 460-nm band is stimulated with UV light at $\lambda_{\mathrm{ex}}<225 \mathrm{~nm}$, while the 410-nm band is induced only at $\lambda_{\mathrm{ex}} \approx 240 \mathrm{~nm}$. For $E / / Z$, the 410 -nm band decreases in intensity, so that one can see only the 460-nm band appreciably.

The 3D spectrum of LGN for $E / / Y$ is shown in Fig. 3. An intense emission band peaking at $500 \mathrm{~nm}$ is excited with UV light at $\lambda_{\mathrm{ex}}=275 \mathrm{~nm}$. The peak position changes to $535 \mathrm{~nm}$ when $\lambda_{\mathrm{ex}}<250 \mathrm{~nm}$. For $E / / Z$, we observed only the 535-nm band. This band is strongly stimulated at $\lambda_{\mathrm{ex}} \approx 275 \mathrm{~nm}$, and exhibits no substantial peak change when $\lambda_{\mathrm{ex}}$ is shortened.

\section{Discussion}

The electronic structure of LGS has been studied experimentally and theoretically [5]. The valence band is mainly formed by the $\mathrm{O} 2 p$ states. The lower part of the conduction band is dominated by the La $5 d$ states, while the upper part is built up of the Ga $4 p$ states with small hybridization with the $\mathrm{Si} 3$ s and $3 p$ states. In LGT, the substitution of $\mathrm{Si}^{4+}$ with $\mathrm{Ga}^{3+}$ and $\mathrm{Ta}^{5+}$ is described as follows: $\left(\mathrm{Si}^{4+}\right)_{\text {tetra }}+0.5\left(\mathrm{Ga}^{3+}\right)_{\text {octa }} \rightarrow\left(\mathrm{Ga}^{3+}\right)_{\text {tetra }}+0.5\left(\mathrm{Ta}^{5+}\right)_{\text {octa }}$, where "tetra" and "octa” mean tetrahedral and octahedral sites, respectively. In LGN, the substitution of $\mathrm{Si}^{4+}$ with $\mathrm{Ga}^{3+}$ and $\mathrm{Nb}^{5+}$ is as follows: $\left(\mathrm{Si}^{4+}\right)_{\text {tetra }}+0.5\left(\mathrm{Ga}^{3+}\right)_{\text {octa }} \rightarrow\left(\mathrm{Ga}^{3+}\right)_{\text {tetra }}+0.5\left(\mathrm{Nb}^{5+}\right)_{\text {octa. }}$ These substitutions will not exert significant influence upon both the valence band composed of the $\mathrm{O}$ $2 p$ states and the lower conduction band of the La $5 d$ states. It is thus expected that the electronic structures responsible for the luminescence processes are basically similar in the langasite family.

In LGS, there exist two emission bands at 420 and $500 \mathrm{~nm}$. The excitation threshold of the 420-nm band coincides with the fundamental absorption edge. Such a coincidence suggests that this band is intrinsic feature of LGS. Since the valence band is built up of the $\mathrm{O} 2 p$ states, it is likely that a hole is self-trapped on an oxygen ion because of the strong interaction with phonons. Therefore, a self-trapped exciton (STE) is formed through the capture of a conduction electron by the self-trapped hole. We suppose that the 420-nm band arises from the 
radiative recombination of STEs. On the other hand, the 500-nm band is stimulated with UV light below the fundamental absorption edge. This band is probably ascribed to a radiative transition in some lattice imperfection. No appreciable changes in intensity and spectral shape between $E / / Y$ and $E / / Z$ were observed for both bands in LGS, which indicates that the LGS luminescence bands are not polarized in the $Y Z$ plane.

In LGT, one can see two emission bands at 410 and $460 \mathrm{~nm}$. The former is intensely induced in the vicinity of the fundamental absorption edge, revealing the extrinsic nature. The latter is excited in wavelength region shorter than the cut-off. This implies that it is attributed to the radiative annihilation of STEs in LGT. The intensity of the 410-nm band is much greater for $E / / Y$ than for $E / / Z$. As mentioned before, the polarization dependence was examined by using two samples with different orientations. The LGT crystals are slightly colored, in contrast to transparent LGS crystals with good quality. We cannot be certain whether the measured polarization is a characteristic feature of the 410-nm luminescence or is due to the sample-dependent intensity change.

When LGN is excited in the wavelength region shorter than $240 \mathrm{~nm}$ for $E / / Y$ or $275 \mathrm{~nm}$ for $E / / Z$, one broad band is observed at $535 \mathrm{~nm}$. This band is probably due to the annihilation of STEs, because the excitation threshold $(275 \mathrm{~nm})$ coincides with the cut-off wavelength. For $E / / Y$, the $500-\mathrm{nm}$ band appears at $\lambda_{\mathrm{ex}} \approx 275 \mathrm{~nm}$, which may be ascribed to perturbed STEs since the LGN crystals are considerably colored.

In the above argument, we assigned the emission bands at $420 \mathrm{~nm}$ (LGS), $460 \mathrm{~nm}$ (LGT), and $535 \mathrm{~nm}$ (LGN) to the luminescence from an STE located on oxygen site in the respective crystals. It is interesting to note that the order of these emission peaks is parallel to that of the fundamental absorption edges: $235 \mathrm{~nm}(\mathrm{LGS})<242 \mathrm{~nm}(\mathrm{LGT})<284 \mathrm{~nm}(\mathrm{LGN})$. Such a fact indicates that the peak energy of the STE luminescence is mainly governed by the band-gap energy. In other words, the Stokes shift due to the lattice relaxation induced by the self-trapping of exciton is nearly equal. This means that the STEs have a similar relaxed configuration in the crystals with langasite structure.

\section{Acknowledgement}

A part of this work was supported by a Grand-in-Aid from the Ministry of Education, Culture, Sports, Science, and Technology of Japan.

\section{References}

[1] B. V. Mill, A. V. Butashin, G. G. Khodzhabagyan, E. L. Belokoneva, N. V. Belov, Dokl. Akad. Nauk SSSR 264 (1982) 1385.

[2] A. N. Gotalskaya, D. I. Drezin, V. V. Bezdelkin, V. N. Stassevich, Proceedings of the 1993 
IEEE International Frequency Control Symposium, edited by G. R. Johnson (Salt Lake City, 1993), p.339.

[3] K. Shimamura, H. Takeda, T. Kohno, T. Fukuda, J. Cryst. Growth 163 (1996) 388.

[4] J. Bohm, R. B. Heimann, M. Hengst, R. Roewer, J. Schindler, J. Cryst. Growth 204 (1999) 128.

[5] M. Kitaura, K. Mochizuki, Y. Inabe, M. Itoh, H. Nakagawa, S. Oishi, Phys. Rev. B 69 (2004) 115120 . 


\section{Figure Captions}

Fig. 1. 3D display of the excitation-emission spectrum of LGS measured for $E / / Y$ at $5 \mathrm{~K}$.

Fig. 2. 3D display of the excitation-emission spectrum of LGT measured for $E / / Y$ at $5 \mathrm{~K}$.

Fig. 3. 3D display of the excitation-emission spectrum of LGN measured for $E / / Y$ at $5 \mathrm{~K}$. 


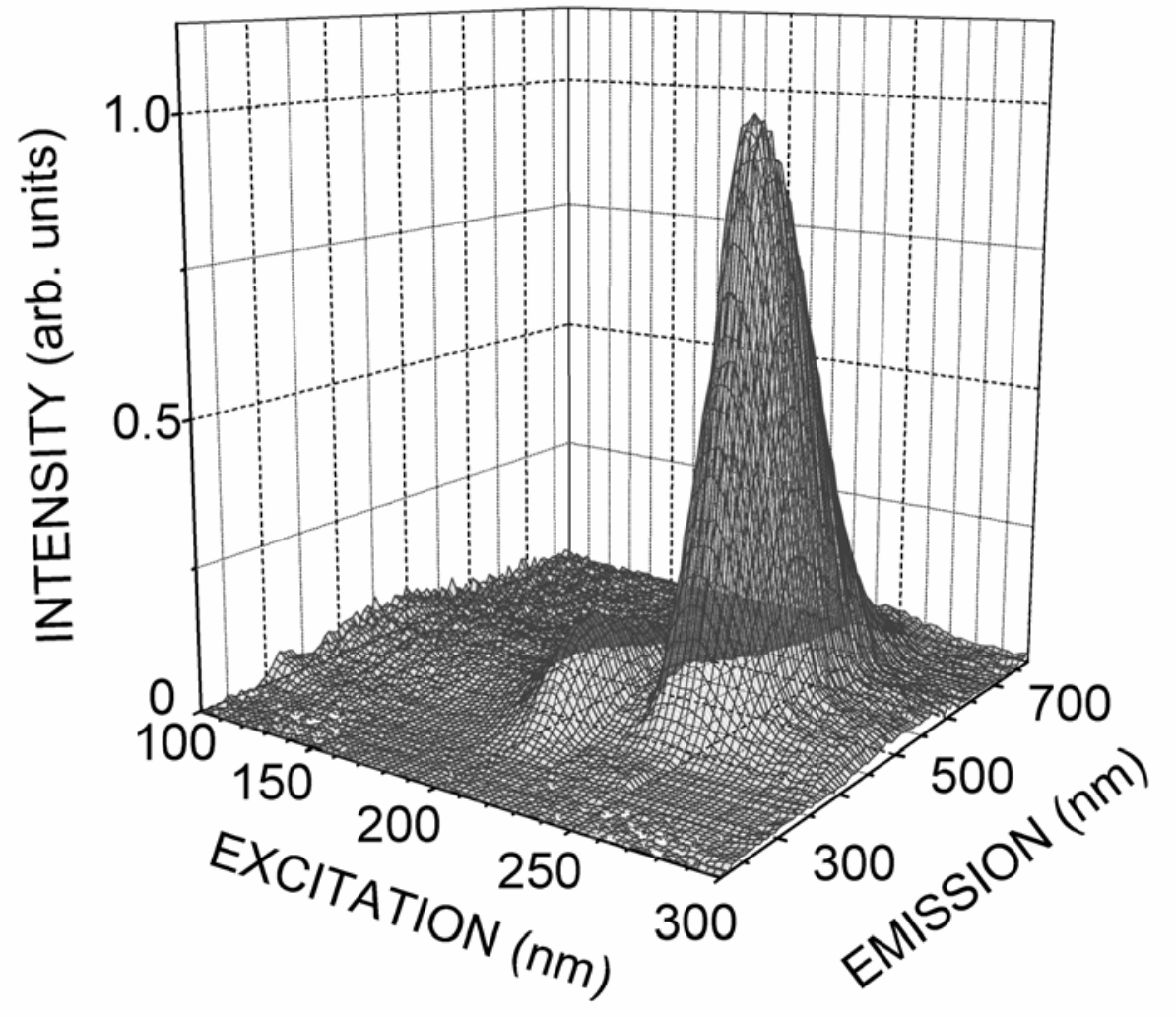

Fig. 1 


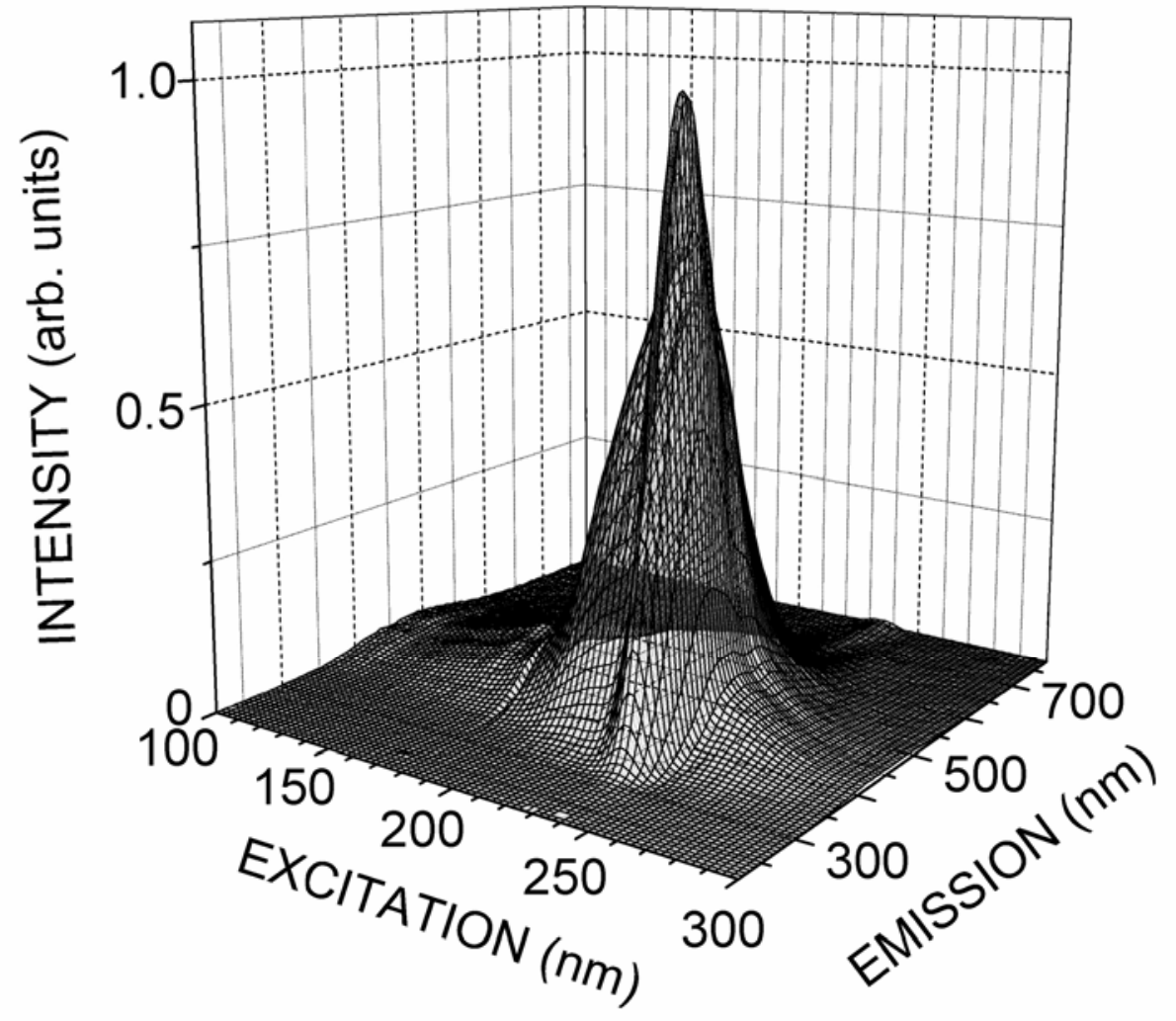

Fig. 2 


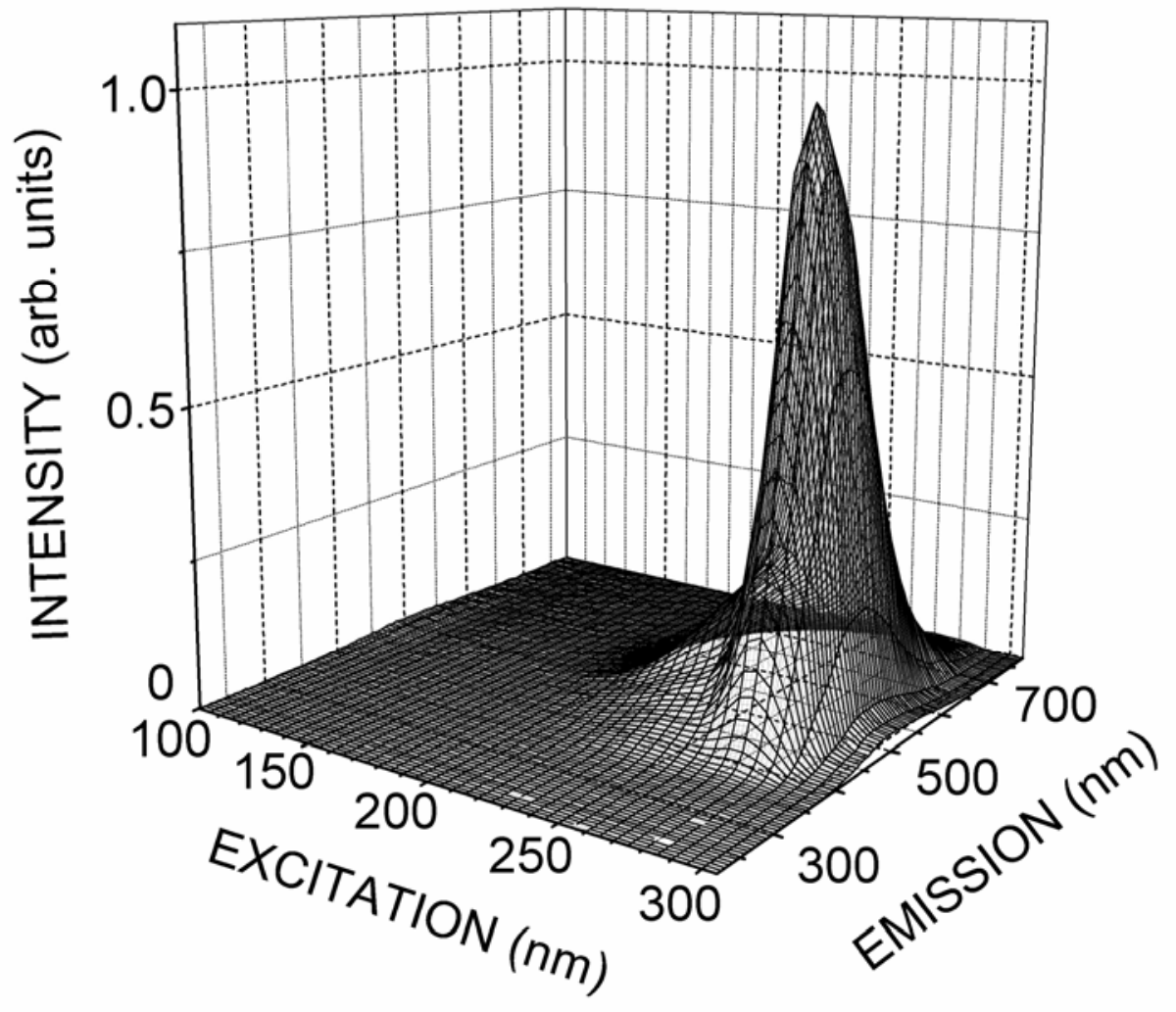

Fig. 3 